\title{
ON RADIAL FOURIER MULTIPLIERS AND ALMOST EVERYWHERE CONVERGENCE
}

\author{
SANGHYUK LEE ANDREAS SEEGER
}

\begin{abstract}
We study a.e. convergence on $L^{p}$, and Lorentz spaces $L^{p, q}$, $p>\frac{2 d}{d-1}$, for variants of Riesz means at the critical index $d\left(\frac{1}{2}-\frac{1}{p}\right)-\frac{1}{2}$. We derive more general results for (quasi-)radial Fourier multipliers and associated maximal functions, acting on $L^{2}$ spaces with power weights, and their interpolation spaces. We also include a characterization of boundedness of such multiplier transformations on weighted $L^{2}$ spaces, and a sharp endpoint bound for Stein's square-function associated with the Riesz means.
\end{abstract}

\section{INTRODUCTION}

Let $\rho \in C^{\infty}\left(\mathbb{R}^{d} \backslash\{0\}\right)$ be a homogeneous distance function of degree $\beta>0$, i.e. $\rho$ satisfies $\rho\left(t^{1 / \beta} \xi\right)=t \rho(\xi)$ for all $t>0$ and $\rho(\xi)>0$ for $\xi \neq 0$. For Schwartz functions $f$ on $\mathbb{R}^{d}$ ( $d \geq 2$ throughout this paper) define the Riesz means $S_{t}^{\lambda} f$ of the Fourier integral by

$$
S_{t}^{\lambda} f(x)=\frac{1}{(2 \pi)^{d}} \int_{\rho(\xi) \leq t}\left(1-\frac{\rho(\xi)}{t}\right)^{\lambda} \widehat{f}(\xi) e^{i\langle x, \xi\rangle} d \xi .
$$

In order to consider almost everywhere convergence on $L^{p}$ one needs to be able to define $S_{t}^{\lambda} f$ as a measurable function, for all $f \in L^{p}\left(\mathbb{R}^{d}\right)$. A necessary condition for $S_{t}^{\lambda}$ to extend as a continuous operator from $L^{p}$ to the space $\mathcal{S}^{\prime}$ of tempered distributions is that the convolution kernel belongs to $L^{p^{\prime}}$ (cf. 5. for a similar comment, and 92 below). This is the case if and only if $\lambda>\lambda(p):=d\left(\frac{1}{2}-\frac{1}{p}\right)-\frac{1}{2}$. In view of the compact support of the multiplier the distribution $S_{t}^{\lambda} f$ is then a bounded $C^{\infty}$ function; moreover the maximal function $\sup _{t>0}\left|S_{t}^{\lambda} f(x)\right|$ is well defined as a measurable function. Carbery, Rubio de Francia and Vega [4] studied the pointwise behavior of $S_{t}^{\lambda}$ and showed that, for $\rho(\xi)=|\xi|$ and $\lambda>\max \{\lambda(p), 0\}$, the means $S_{t}^{\lambda} f(x)$ converge almost everywhere to $f(x)$. The method in [4] is based on the trace theorem for Sobolev functions and applies to the general situation considered here, see also Sato [17]. For an extension involving nonisotropic distance functions see Cladek [7].

1991 Mathematics Subject Classification. 42B15, 42B25.

Key words and phrases. Square functions, Riesz means, maximal Bochner-Riesz operator, radial multipliers, quasiradial multipliers, Herz spaces, Lorentz spaces.

Supported in part by NRF grant 2012008373 and NSF grant 1200261. 
In this paper we investigate what happens at the critical index $\lambda=\lambda(p)=$ $d\left(\frac{1}{2}-\frac{1}{p}\right)-\frac{1}{2}$ when $\lambda(p)>0$, i.e. when $p>\frac{2 d}{d-1}$. It is natural either to consider $S_{t}^{\lambda}$ on smaller Lorentz spaces, or to slightly regularize the multiplier to produce an operator well defined on $L^{p}\left(\mathbb{R}^{d}\right)$. Let

$$
h_{\lambda, \gamma}(r)=\frac{(1-r)_{+}^{\lambda}}{\left(1+\log \left(\frac{1}{1-r}\right)\right)^{\gamma}} .
$$

Define the generalized Bochner Riesz means $S_{t}^{\lambda, \gamma} f$ by

$$
\widehat{S_{t}^{\lambda, \gamma} f}(\xi)=h_{\lambda, \gamma}\left(\frac{\rho(\xi)}{t}\right) \widehat{f}(\xi) .
$$

For the critical $\lambda=\lambda(p)$ these operators extend to bounded operators from the Lorentz space $L^{p, q}$ to $\mathcal{S}^{\prime}$ if and only if $\gamma>1-1 / q$ when $q>1$ and $\gamma \geq 0$ when $q=1$; cf. 2 . In the thesis [1] Annoni showed, for $\rho(\xi)=|\xi|$, that $S_{t}^{\lambda(p), \gamma} f(x) \rightarrow f(x)$ a.e. for all $f \in L^{p}, p>\frac{2 d}{d-1}$ under the condition $\gamma>3 / 2-1 / p$. This left open the range $1-1 / p<\gamma \leq 3 / 2-1 / p$. Almost everywhere convergence in this range is implied by the case $q=p$ of the following theorem.

Theorem 1.1. Let $\frac{2 d}{d-1}<p<\infty, \lambda(p)=d\left(\frac{1}{2}-\frac{1}{p}\right)-\frac{1}{2}$. Let $\gamma>1-\frac{1}{q}$, $1<q \leq \infty$, or $\gamma \geq 0$ and $q=1$. Then for $f \in L^{p, q}$ we have

$$
\lim _{t \rightarrow \infty} S_{t}^{\lambda(p), \gamma} f(x)=f(x) \text { a.e. }
$$

Note that for $q=1$ this covers an endpoint result for the Riesz means.

The usual approach to pointwise convergence results for functions in $L^{p, q}\left(\mathbb{R}^{d}\right)$ is to prove an $L^{p, q} \rightarrow L^{p, \infty}$ bound for the associated maximal function $S_{*}^{\lambda, \gamma} f=\sup _{t>0}\left|S_{t}^{\lambda, \gamma} f\right|$. By Stein's theorem [21] such an estimate is necessary for $p \leq 2$, see Tao 22 for what is currently known for the maximal Bochner-Riesz operator in that range. For $p>2$ complete $L^{p_{-}}$ boundedness results for the maximal Bochner-Riesz operator $(\lambda>\lambda(p))$ are known in two dimensions ([3]) and partial results have been proved in higher dimensions $([6],[18,[13],[15])$. For the endpoint $\lambda=\lambda(p)$ sharp results were recently observed in [14], for the case $\rho(\xi)=|\xi|$, in the restricted range $\frac{2 d+2}{d-1}<p<\infty$. For these parameters $S_{*}^{\lambda(p), 0}$ maps $L^{p, 1}$ to $L^{p}$ and $S_{*}^{\lambda(p), \gamma} \operatorname{maps} L^{p, q}$ to $L^{p}$ if $q \leq p$ and $\gamma>1-1 / q$. We note that the sharp $L^{p, 1} \rightarrow L^{p}$ endpoint bounds for the maximal operator are not known in the range $\frac{2 d}{d-1}<p \leq \frac{2(d+1)}{d-1}$, even in two dimensions. Still less is known for general $\rho$. Thus we opt for a variant of the approach by Carbery, Rubio de Francia and Vega [4], who used weighted $L^{2}\left(|x|^{-a} d x\right)$ spaces. Annoni [1] followed this approach and introduced logarithmic modifications of the weight function, working with $|x|^{-a}(\log (2+|x|))^{-\mu}$ for suitable $\mu>0$. We prefer to preserve the homogeneity of the weight and use the observation that for $p>2$ the space $L^{p, 2}$ is embedded in $L^{2}\left(|x|^{-d(1-2 / p)} d x\right)$. We are able to sharpen the analysis in [4] to prove boundedness in this space for maximal 
operators defined by $\sup _{t>0}\left|\mathcal{F}^{-1}\left[h\left(\frac{\rho(\cdot)}{t}\right) \widehat{f}\right]\right|$ where $h$ is supported in $\left[\frac{1}{2}, 2\right]$ and belongs to the $L^{2}$-Sobolev space $\mathcal{L}_{\alpha}^{2} \equiv B_{\alpha, 2}^{2}$ with $\alpha=d(1 / 2-1 / p)$. This will be a special case of Theorem 1.2 below and can also be deduced from the square-function estimate in Theorem 1.5. In particular for the range $\gamma>1 / 2$ the maximal operator $S_{*}^{\lambda(p), \gamma}$ is bounded on $L^{2}\left(|x|^{-d(1-2 / p)} d x\right)$; this implies the $q=2$ case of Theorem 1.1 .

In order to get a complete result for all $q$ (in particular $q=p$ ) it is convenient to work with the homogeneous Herz spaces $\dot{\mathcal{K}}_{r}^{\gamma, r}$, in the special case $r=2$. For fixed $r$ these are real interpolation spaces of the $L^{r}\left(|x|^{r \gamma} d x\right)$ spaces, see Gilbert 11]. The definition (following the terminology in [2]) is as follows. Let, for $l \in \mathbb{Z}, \mathfrak{A}_{l}:=\left\{x: 2^{l} \leq|x|<2^{l+1}\right\}$. Then $\dot{\mathcal{K}}_{r}^{\gamma, q}$ is the space of all functions which are $r$-integrable on compact subsets of $\mathbb{R}^{d} \backslash\{0\}$ and for which

$$
\|f\|_{\dot{\mathcal{K}}_{r}^{\gamma, q}}=\left(\sum_{l \in \mathbb{Z}} 2^{l \gamma q}\left[\int_{\mathfrak{A}_{l}}|f(x)|^{r} d x\right]^{q / r}\right)^{1 / q}
$$

is finite. It is easy to see that for $\gamma>-d / r$ every function in $\dot{\mathcal{K}}_{r}^{\gamma, q}$ defines a unique tempered distribution on $\mathcal{S}^{\prime}\left(\mathbb{R}^{d}\right)$. In what follows we use the notation $\lesssim$ for an inequality which involves an implicit constant.

Theorem 1.2. Let $\frac{1}{2}<\alpha<\frac{d}{2}, 1 \leq q \leq \infty$ and $s=\frac{q}{q-1}$. Let $h \in B_{\alpha, s}^{2}(\mathbb{R})$ be supported in $\left(\frac{1}{2}, 2\right)$. Then the following hold.

(i) For $2 \leq q \leq \infty$,

$$
\left\|\sup _{t>0} \mid \mathcal{F}^{-1}\left[h\left(\frac{\rho(\cdot)}{t}\right) \widehat{f}\right]\right\|_{\dot{\mathcal{K}}_{2}^{-\alpha, q}} \lesssim\|h\|_{B_{\alpha, s}^{2}}\|f\|_{\dot{\mathcal{K}}_{2}^{-\alpha, q}}
$$

(ii) For $1 \leq q \leq 2$,

$$
\left\|\sup _{t>0} \mid \mathcal{F}^{-1}\left[h\left(\frac{\rho(\cdot)}{t}\right) \widehat{f}\right]\right\|_{\dot{\mathcal{K}}_{2}^{-\alpha, 2}} \lesssim\|h\|_{B_{\alpha, s}^{2}}\|f\|_{\mathcal{K}_{2}^{-\alpha, q}} .
$$

For $p>2$ and $\alpha=d(1 / 2-1 / p)$ there is the embedding $L^{p, q} \subset \dot{\mathcal{K}}_{2}^{-\alpha, q},(c f$. Lemma 3.1 below). By a standard approximation argument we will show

Corollary 1.3. Let $p>\frac{2 d}{d-1}, 1 \leq q \leq \infty, s=\frac{q}{q-1}$, and let $h \in B_{d\left(\frac{1}{2}-\frac{1}{p}\right), s}^{2}$ be supported in a compact subinterval of $(0, \infty)$. Then for $f \in L^{p, q}$

$$
\lim _{t \rightarrow \infty}\left|\mathcal{F}^{-1}[h(\rho / t) \widehat{f}](x)\right|=0 \text { a.e. }
$$

If $\chi \in C_{0}^{\infty}(\mathbb{R})$ is compactly supported away from the origin then $\chi h_{\lambda, \gamma} \in$ $B_{\alpha, s}^{2}$ for $\alpha=\lambda+1 / 2$ and $\gamma>1 / s$, and $\gamma \geq 0$ if $s=\infty$, see e.g. [23]. Thus one can deduce Theorem 1.1 from Corollary 1.3 .

The proof of Theorem 1.2 for $q=2$ also gives a characterization of boundedness convolution operators with quasiradial multipliers on weighted $L^{2}$ spaces with power weights (i.e. on the spaces $\dot{\mathcal{K}}_{ \pm \alpha, 2}^{2}$ ). The result is in the spirit of (but in some sense complementary to) the results by Muckenhoupt, Wheeden and Young [16]. In what follows let $\varphi \in C_{0}^{\infty}\left(\frac{1}{2}, 2\right)$ be a nontrivial bump function. 
Theorem 1.4. Let $\rho$ be as above and let $1 / 2<\alpha<d / 2$. Define the operator $T$ by $\widehat{T f}(\xi)=m(\rho(\xi)) \widehat{f}(\xi)$. Then the following statements are equivalent:

(i) $T$ is bounded on $L^{2}\left(|x|^{-2 \alpha} d x\right)$.

(ii) $T$ is bounded on $L^{2}\left(|x|^{2 \alpha} d x\right)$.

(iii) $\sup _{t>0}\|\varphi m(t \cdot)\|_{L_{\alpha}^{2}(\mathbb{R})}<\infty$.

Finally we prove a sharp bound for Stein's square-function associated with the Riesz means,

$$
G_{\alpha} f(x)=\left(\int_{0}^{\infty}\left|S_{t}^{\alpha-1} f(x)-S_{t}^{\alpha} f(x)\right|^{2} \frac{d t}{t}\right)^{1 / 2}
$$

which can also be used to prove Theorem 1.4 ,

Theorem 1.5. Let $\frac{1}{2}<\alpha<\frac{d}{2}$. Then for $f \in L^{2}\left(|x|^{-2 \alpha} d x\right)$,

$$
\int\left|G_{\alpha} f(x)\right|^{2} \frac{d x}{|x|^{2 \alpha}} \lesssim \int|f(x)|^{2} \frac{d x}{|x|^{2 \alpha}}
$$

This paper. In $\ 2$ we discuss the definition of our multiplier transformations and associated maximal functions on Lebesgue and Lorentz spaces, and then prove a convenient characterization of quasiradial multipliers to be Fourier transforms of functions in these spaces (see Theorem 2.2). In the preliminary section 93 we consider embeddings for Lorentz and Herz spaces which are needed to deduce Theorem 1.1 from Corollary 1.3. We also review the Fourier restriction estimate in weighted $L^{2}$ spaces, use it to prove a basic maximal function estimate, and discuss other basic preliminaries. The main section $\$ 4$ contains the proof of Theorem 1.2. The proofs of Theorem 1.4 and Corollary 1.3 are given in $\$ 5$, and Theorem 1.5 is proved in $\$ 6$.

Acknowledgement. The authors would like to thank Jong-Guk Bak for conversations at the early stages of this work.

\section{Definition of CONVOLUtion AND MAXimal OPERATORS ON $L^{p, q}$}

For $f \in L^{p}$ the expression $S_{t}^{\lambda, \gamma} f$ is not necessarily defined for all $f \in L^{p, q}$, even in the sense of tempered distributions, since the Fourier transform of an $L^{p}$ function does not have to be a function.

Lemma 2.1. Suppose $1 \leq p, q<\infty$. Let $K$ be a tempered distribution in $\mathbb{R}^{d}$ such that $\widehat{K}$ has compact support. For $f \in \mathcal{S}\left(\mathbb{R}^{d}\right)$ define $T_{K}=f * K$. Then

(i) $T_{K}$ extends to a continuous linear operator from $L^{p, q}$ to $\mathcal{S}^{\prime}$ if and only if $K \in L^{p^{\prime}, q^{\prime}}$.

(ii) If $K \in L^{p^{\prime}, q^{\prime}}$ then for $f \in L^{p, q}$ the maximal function

$$
\sup _{t>0}\left|t^{d} K(t \cdot) * f(x)\right|
$$

is Borel measurable. 
Proof. Assume that $T_{K}: L^{p, q} \rightarrow \mathcal{S}^{\prime}$ is continuous. Then for every $g \in \mathcal{S}$ there is a constant $C(g)$ such that $\left|\left\langle T_{K} f, g\right\rangle\right| \leq C(g)\|f\|_{L^{p, q}}$. Observe that if $g \in \mathcal{S}$ such that $\widehat{g}(\xi)=1$ for $\xi \in \operatorname{supp}(\widehat{K})$ then $\langle K * f, g\rangle=\langle f, K(-\cdot)\rangle$. Thus $K$ must lie in the dual space of $L^{p, q}$, i.e. in $L^{p^{\prime}, q^{\prime}}$. Conversely, if $K \in K^{p^{\prime}, q^{\prime}}$ then for every $f \in L^{p, q}$ the convolution $x \mapsto K * f(x)$ is well defined as a bounded continuous function. This shows $(i)$. Since the supremum of continuous functions is Borel measurable we get $(i i)$.

Quasiradial functions in $\mathcal{F} L^{u, s}$. We consider $m=h \circ \rho$ where $\rho$ is a homogeneous distance function of degree $\beta$, and $\rho \in C^{\infty}\left(\mathbb{R}^{d} \backslash\{0\}\right)$. It is useful to express the condition $(i i)$ in Lemma 2.1 in terms of the onedimensional Fourier transform of $h_{\beta}(t)=h\left(t^{\beta}\right)$. The following theorem sharpens a result in [19] for the class of distance functions considered here; the radial analogue, for $\rho(\xi)=|\xi|$, is already in [9]. Note that here we make no curvature assumption on the $\rho$-unit sphere

$$
\Sigma_{\rho}=\{\xi: \rho(\xi)=1\} .
$$

The following result may be interesting in its own right but we shall use it only to demonstrate sharpness of our results; it is not needed in the proofs of Theorems 1.2, 1.5 .

Theorem 2.2. Let $h$ be supported in a compact subinterval $J$ of $(0, \infty)$ and let

$$
\mathcal{K}_{\beta}(r)=\frac{1}{2 \pi} \int h\left(t^{\beta}\right) e^{i r t} d t .
$$

Let $1<u<2,1 \leq s \leq \infty$ and $\mu_{d}$ be the measure on $\mathbb{R}$ given by

$$
d \mu_{d}(r)=(1+|r|)^{d-1} d r .
$$

Then

$$
\left\|\mathcal{F}^{-1}[h \circ \rho]\right\|_{L^{u, s\left(\mathbb{R}^{d}\right)}} \approx\left\|\frac{\mathcal{K}_{\beta}}{(1+|\cdot|)^{\frac{d-1}{2}}}\right\|_{L^{u, s}\left(\mathbb{R}, \mu_{d}\right)} .
$$

For $u=s$ this equivalence becomes

$$
\left\|\mathcal{F}^{-1}[h \circ \rho]\right\|_{L^{u}\left(\mathbb{R}^{d}\right)} \approx\left(\int\left|\mathcal{K}_{\beta}(r)\right|^{u}(1+|r|)^{(d-1)\left(1-\frac{u}{2}\right)} d r\right)^{1 / u} .
$$

In the proof of Theorem 2.2 we shall use an elementary convolution inequality in weighted spaces.

Lemma 2.3. For $a \in \mathbb{R}$ let $M_{a}$ be the multiplication operator $M_{a} g(r)=$ $g(r)(1+|r|)^{a}$. Let $\varsigma$ be a measurable function such that for all $N$

$$
|\varsigma(r)| \leq C(N)(1+|r|)^{-N} .
$$

Then for all $a \in \mathbb{R}, 1 \leq u \leq \infty$

$$
\left\|M_{-a}\left[\varsigma *\left(M_{a} g\right)\right]\right\|_{L^{u, s}\left(\mu_{d}\right)} \lesssim\|g\|_{L^{u, s}\left(\mu_{d}\right)} .
$$


Proof. The straightforward proof is left to the reader. Real interpolation reduces this to the case $u=s$ for which one can consult Lemma 2.2 in [9].

Proof of Theorem 2.2. Since $\rho^{1 / \beta}$ is homogeneous of degree 1 the statement follows immediately from the special case $\beta=1$, which we will henceforth assume. We shall write $\kappa$ for $\mathcal{K}_{1}$, so that $\kappa=\mathcal{F}_{\mathbb{R}}^{-1}[h]$.

Proof of $\lesssim$ in (3). Let $\chi_{1}$ in $C^{\infty}$, compactly supported in $(0, \infty)$ such that $\chi_{1}(\rho)=1$ on the support of $h$. Since $\kappa=\widehat{h}$ it suffices to show

$$
\left\|\mathcal{F}^{-1}\left[\chi_{1} \circ \rho \widehat{\kappa} \circ \rho\right]\right\|_{L^{u, s}\left(\mathbb{R}^{d}\right)} \lesssim\left\|(1+|\cdot|)^{-\frac{d-1}{2}} \kappa\right\|_{L^{u, s}\left(\mu_{d}\right)}
$$

which follows from

$$
\left\|\mathcal{F}^{-1}\left[\chi_{1} \circ \rho \int g(r)(1+|r|)^{\frac{d-1}{2}} e^{-i r \rho} d r\right]\right\|_{L^{u, s}\left(\mathbb{R}^{d}\right)} \lesssim\|g\|_{L^{u, s}\left(\mu_{d}\right)} .
$$

We show the corresponding inequalities with $L^{u, s}$ replaced by $L^{1}$ and by $L^{2}$.

For the $L^{1}$ inequality we use that

$$
\left\|\mathcal{F}^{-1}\left[\chi_{1} \circ \rho e^{-i r \rho}\right]\right\|_{L^{1}\left(\mathbb{R}^{d}\right)} \lesssim(1+|r|)^{\frac{d-1}{2}}
$$

which is a rescaled inequality from [20]. Here it is crucial that $\rho$ is homogeneous of degree one. By Minkowski's inequality the displayed estimate yields

$$
\left\|\mathcal{F}^{-1}\left[\chi_{1} \circ \rho \int g(r)(1+|r|)^{\frac{d-1}{2}} e^{-i r \rho} d r\right]\right\|_{L^{1}\left(\mathbb{R}^{d}\right)} \lesssim \int|g(r)|(1+|r|)^{d-1} d r
$$

For the $L^{2}$ inequality we use polar coordinates and Plancherel's theorem in $\mathbb{R}^{d}$ and $\mathbb{R}$, to get

$$
\begin{aligned}
& \left\|\mathcal{F}^{-1}\left[\chi_{1} \circ \rho \int g(r)(1+|r|)^{\frac{d-1}{2}} e^{-i r \rho} d r\right]\right\|_{L^{2}\left(\mathbb{R}^{d}\right)} \\
& \lesssim\left\|\chi_{1} \circ \rho(\cdot) \int g(r)(1+|r|)^{\frac{d-1}{2}} e^{-i r \rho(\cdot)} d r\right\|_{L^{2}\left(\mathbb{R}^{d}\right)} \\
& \lesssim\left\|\chi_{1} \int g(r)(1+|r|)^{\frac{d-1}{2}} e^{-i r(\cdot)} d r\right\|_{L^{2}(\mathbb{R})} \\
& \lesssim\left(\int|g(r)|^{2}(1+|r|)^{d-1} d r\right)^{1 / 2} .
\end{aligned}
$$

The asserted inequality (41) follows from (5) and (6) by real interpolation.

Proof of $\gtrsim$ in (3). We shall work with smooth $h$ which allows to assume that the $L^{u, s}\left(\mu_{d}\right)$ norm of $(1+|r|)^{-\frac{d-1}{2}} \kappa(r)$ is a priori finite. With this assumption we have to prove the inequality

$$
\left\|(1+|\cdot|)^{-(d-1) / 2} \kappa\right\|_{L^{u, s}\left(\mu_{d}\right)} \lesssim\left\|\mathcal{F}^{-1}[h \circ \rho]\right\|_{L^{u, s}\left(\mathbb{R}^{d}\right)} .
$$

If (17) holds for all smooth $h$ supported in $J$ then the general case can be derived by an approximation argument. 
Pick $\xi_{0} \in \Sigma_{\rho}$ so that $|\xi|$ has a maximum at $\xi_{0}$. Then the Gauss map is injective in a small neighborhood $U$ on the surface $\Sigma_{\rho}$ and the curvature is bounded below on $U$. Let $\gamma$ be homogeneous of degree zero, $\gamma\left(\xi_{0}\right) \neq 0$ and supported on the closure of the cone generated by $U$. Clearly

$$
\left\|\mathcal{F}^{-1}[\gamma h \circ \rho]\right\|_{L^{u, s}} \lesssim\left\|\mathcal{F}^{-1}[h \circ \rho]\right\|_{L^{u, s}}
$$

Now use $\rho$ polar coordinates to write

$$
\mathcal{F}^{-1}[\gamma h \circ \rho](x)=(2 \pi)^{-d} \int_{0}^{\infty} h(\rho) \rho^{d-1} \int_{\Sigma_{\rho}} \gamma\left(\xi^{\prime}\right) e^{i \rho\left\langle\xi^{\prime}, x\right\rangle} \frac{d \sigma\left(\xi^{\prime}\right)}{\left|\nabla \rho\left(\xi^{\prime}\right)\right|} d \rho .
$$

Let $n\left(\xi_{0}\right)$ the outer normal at $\xi_{0}$, let $\Gamma=\left\{x \in \mathbb{R}^{d}:\left|\frac{x}{|x|}-n\left(\xi_{0}\right)\right| \leq \varepsilon\right\}$, with $\varepsilon$ small and let, for large $R \gg 1, \Gamma_{R}=\{x \in \Gamma:|x| \geq R\}$. By choosing $\varepsilon$ small enough we may assume that for each $x \in \Gamma$ there is a unique $\xi=\Xi(x) \in \Sigma_{\rho}$, so that $\gamma(\Xi(x)) \neq 0$ and so that $x$ is normal to $\Sigma_{\rho}$ at $\Xi(x)$. Clearly $x \mapsto \Xi(x)$ is homogeneous of degree zero on $\Gamma$. By the method of stationary phase we have for $x \in \Gamma_{R}$

$$
\mathcal{F}^{-1}[\gamma h(\rho(\cdot))](x)=I_{0}(x)+\sum_{j=1}^{N} I I_{j}(x)+I I I(x)
$$

where

$$
I_{0}(x)=c \int_{0}^{\infty} h(\rho) \rho^{\frac{d-1}{2}} e^{i \rho\langle\Xi(x), x\rangle} d \rho \frac{\gamma(\Xi(x))|\nabla \rho(\Xi(x))|^{-1}}{(\langle\Xi(x), x\rangle)^{\frac{d-1}{2}}|K(\Xi(x))|^{1 / 2}},
$$

$|c|=(2 \pi)^{-d}$ and $K(\Xi(x))$ is the curvature of $\Sigma_{\rho}$ at $\Xi(x)$. There are similar formulas for the higher order terms $I I_{j}(x)$ with the main term $(\rho\langle\Xi(x), x\rangle)^{-\frac{d-1}{2}}$ replaced by $(\rho\langle\Xi(x), x\rangle)^{-\frac{d-1}{2}-j}$. Finally

$$
|I I I(x)| \lesssim_{N}\|h\|_{1}|x|^{-N}, \quad x \in \Gamma_{R} .
$$

Let $h_{j}(\rho)=h(\rho) \rho^{\frac{d-1}{2}-j}$ and let $\kappa_{j}=\mathcal{F}_{\mathbb{R}}^{-1}\left[h_{j}\right]$, then, for some $C_{1} \geq 1$,

$$
C_{1}^{-1}\left|I_{0}(x)\right| \leq \frac{|\kappa(\langle\Xi(x), x\rangle)|}{\mid\langle\Xi(x) x, \mid\rangle^{\frac{d-1}{2}}} \leq C_{1}\left|I_{0}(x)\right|, \quad x \in \Gamma_{R} .
$$

We also have, for some $C_{0} \geq 1$,

$$
C_{0}^{-1}|x| \leq\langle\Xi(x), x\rangle \leq C_{0}|x|, \quad x \in \Gamma,
$$

which is a consequence of Euler's homogeneity relation $\rho(\xi)=\langle\xi, \nabla \rho(\xi)\rangle$ and the positivity assumption on $\rho$. Let

$$
\begin{aligned}
E_{R}(\theta, \alpha) & =\left\{r:|r| \geq R,\left|I_{0}(r \theta)\right|>\alpha\right\}, \\
E_{R}^{*}(\beta) & =\left\{r:|r| \geq C_{0} R: r^{-\frac{d-1}{2}}\left|\kappa_{0}(r)\right|>C_{1} \beta\right\} .
\end{aligned}
$$


Then

$$
\begin{aligned}
\left\|I_{0}\right\|_{L^{u, s}\left(\Gamma_{R}\right)} & \gtrsim\left(\int_{0}^{\infty}\left[\alpha\left(\operatorname{meas}\left\{x \in \Gamma_{R}:\left|I_{0}(x)\right|>\alpha\right\}\right)^{1 / u}\right]^{s} \frac{d \alpha}{\alpha}\right)^{1 / s} \\
& \gtrsim\left(\int_{0}^{\infty}\left[\alpha\left(\int_{S^{d-1} \cap \Gamma} \int_{E_{R}(\theta, \alpha)} r^{d-1} d r d \theta\right)^{1 / u}\right]^{s} \frac{d \alpha}{\alpha}\right)^{1 / s} \\
& \gtrsim\left(\int_{0}^{\infty}\left[\alpha\left(\int_{E_{R}^{*}\left(2^{d+1} \alpha\right)} r^{d-1} d r\right)^{1 / u}\right]^{s} \frac{d \alpha}{\alpha}\right)^{1 / s},
\end{aligned}
$$

which gives

$$
\left\|(1+|\cdot|)^{-\frac{d-1}{2}} \kappa_{0}\right\|_{L^{u, s}\left(\left[C_{0} R, \infty\right], \mu_{d}\right)} \lesssim\left\|I_{0}\right\|_{L^{u, s}\left(\Gamma_{R}\right)} .
$$

A variant of this argument also yields the upper bound

$$
\left\|I_{0}\right\|_{L^{u, s}\left(\Gamma_{R}\right)} \lesssim\left\|(1+|\cdot|)^{-\frac{d-1}{2}} \kappa_{0}\right\|_{L^{u, s}\left(\mu_{d}\right)},
$$

and similarly, taking into account the additional decay in the terms $I I_{j}$,

$$
\begin{aligned}
\left\|I I_{j}\right\|_{L^{u, s}\left(\Gamma_{R}\right)} & \lesssim R^{-1}\left\|(1+|\cdot|)^{-\frac{d-1}{2}} \kappa_{j}\right\|_{L^{u, s}\left(\mu_{d}\right)} \\
& \lesssim R^{-1}\left\|(1+|\cdot|)^{-\frac{d-1}{2}} \kappa_{0}\right\|_{L^{u, s}\left(\mu_{d}\right)} .
\end{aligned}
$$

Here, for the second inequality, we have used Lemma 2.3 and the fact that $\kappa_{j}=\kappa * \varsigma_{j}$ for some Schwartz function $\varsigma_{j}$. We also have the trivial inequalities which use the support properties of $h$

$$
\|I I I\|_{L^{u, s}\left(\Gamma_{R}\right)} \lesssim R^{-1}\|h\|_{1}
$$

and $\|h\|_{1} \lesssim\|h \circ \rho\|_{L^{u^{\prime}, s\left(\mathbb{R}^{d}\right)}} \lesssim\left\|\mathcal{F}^{-1}[h \circ \rho]\right\|_{L^{u, s}\left(\mathbb{R}^{d}\right)}$, by the Hausdorff-Young inequality. Thus

$$
\|I I I\|_{L^{u, s}\left(\Gamma_{R}\right)} \lesssim R^{-1}\left\|\mathcal{F}^{-1}[h \circ \rho]\right\|_{L^{u, s}\left(\mathbb{R}^{d}\right)} .
$$

This argument also gives

$$
\left\|(1+|\cdot|)^{-\frac{d-1}{2}} \kappa_{0}\right\|_{L^{u, s}\left(\left[0, C_{0}\right], \mu_{d}\right)} \lesssim R^{d}\left\|\mathcal{F}^{-1}[h \circ \rho]\right\|_{L^{u, s}}
$$

since the left hand side is estimated by $R^{d}\left\|\kappa_{0}\right\|_{\infty} \lesssim R^{d}\|h\|_{1}$.

We now combine the estimates to show (7). By (10) and (13) we get

$$
\left\|(1+|\cdot|)^{-\frac{d-1}{2}} \kappa_{0}\right\|_{L^{u, s}\left(\mu_{d}\right)} \lesssim\left\|I_{0}\right\|_{L^{u, s}\left(\Gamma_{R}\right)}+R^{d}\left\|\mathcal{F}^{-1}[h \circ \rho]\right\|_{L^{u, s}\left(\mathbb{R}^{d}\right)} .
$$

Since $\left\|\mathcal{F}^{-1}[\gamma h \circ \rho]\right\|_{L^{u, s}\left(\mathbb{R}^{d}\right)} \lesssim\left\|\mathcal{F}^{-1}[h \circ \rho]\right\|_{L^{u, s}\left(\mathbb{R}^{d}\right)}$, by (91), (12) and (11)

$$
\begin{aligned}
& \left\|I_{0}\right\|_{L^{u, s}\left(\Gamma_{R}\right)} \lesssim\left(1+R^{d}\right)\left\|\mathcal{F}^{-1}[h \circ \rho]\right\|_{L^{u, s}\left(\mathbb{R}^{d}\right)}+\sum_{j=1}^{N}\left\|I I_{j}\right\|_{L^{u, s}\left(\Gamma_{R}\right)} \\
& \lesssim\left(1+R^{d}\right)\left\|\mathcal{F}^{-1}[h \circ \rho]\right\|_{L^{u, s}\left(\mathbb{R}^{d}\right)}+R^{-1}\left\|(1+|\cdot|)^{-\frac{d-1}{2}} \kappa_{0}\right\|_{L^{u, s}\left(\mu_{d}\right)} .
\end{aligned}
$$

Hence, combining these two estimates and choosing $R \gg 1$ sufficiently large we get

$$
\left\|(1+|\cdot|)^{-\frac{d-1}{2}} \kappa_{0}\right\|_{L^{u, s}\left(\mu_{d}\right)} \lesssim\left\|\mathcal{F}^{-1}[h \circ \rho]\right\|_{L^{u, s}\left(\mathbb{R}^{d}\right)}
$$


Finally observe that $\kappa=\kappa_{0} * \varsigma_{0}$ for some Schwartz function $\varsigma_{0}$ and thus by Lemma 2.3

$$
\left\|(1+|\cdot|)^{-\frac{d-1}{2}} \kappa\right\|_{L^{u, s}\left(\mu_{d}\right)} \lesssim\left\|(1+|\cdot|)^{-\frac{d-1}{2}} \kappa_{0}\right\|_{L^{u, s}\left(\mu_{d}\right)} .
$$

The desired estimate (7D) follows.

Quasiradial functions in Besov spaces. If $1<u<2$ we have the embedding $\dot{\mathcal{K}}_{2}^{\alpha, s} \hookrightarrow L^{u, s}$ for $\alpha=d(1 / u-1 / 2)$. This holds for $u=1$, $s=1$ by using the Cauchy-Schwarz inequality on each annulus $\mathfrak{A}_{l}$, and it trivially holds for $u=s=2$. We use interpolation ([11]) together with the reiteration theorem for real interpolation to deduce that the embedding holds for $1<u<2$ and all $s>0$.

Next observe that the homogeneous Besov space $\dot{B}_{\alpha, s}^{2}$ is the image of $\dot{\mathcal{K}}_{2}^{\alpha, s}$ under the Fourier transform. If $h$ is supported in a compact subinterval $J$ of $(0, \infty)$ then for $\alpha>0$,

$$
\|h \circ \rho\|_{B_{\alpha, q}^{2}\left(\mathbb{R}^{d}\right)} \lesssim\|h\|_{B_{\alpha, q}^{2}(\mathbb{R})} \lesssim\|h\|_{\dot{B}_{\alpha, q}^{2}(\mathbb{R})} ;
$$

here the implicit constants depend on $J$. Hence we see that if $p>2$ and $h \in B_{d(1 / 2-1 / p), q^{\prime}}^{2}$ is supported in $J$ then $\mathcal{F}^{-1}[h(\rho) \widehat{f}]$ and the associated maximal function $\sup _{t>0} \mid \mathcal{F}^{-1}[h(\rho / t) \widehat{f}]$ are well defined for $f \in L^{p, q}$.

Multipliers of Bochner-Riesz type. Now let $\left\{\eta_{j}\right\}$ be a family of $C^{\infty}$ functions supported on $(1 / 4,1 / 2)$ so that the $C^{10 d}$ norms are uniformly bounded. Define, for sequences $\mathfrak{a}=\left\{a_{j}\right\}_{j=2}^{\infty}$

$$
h[\mathfrak{a}](\tau)=\sum_{j=2}^{\infty} a_{j} 2^{-j \lambda} \eta_{j}\left(2^{j}(1-\tau)\right) .
$$

Then it is easy to see that for $\lambda>-1 / 2$

$$
\|h[\mathfrak{a}]\|_{B_{\lambda+\frac{1}{2}, s}^{2}} \lesssim\|\mathfrak{a}\|_{\ell_{s}} .
$$

Now consider $h_{\lambda, \gamma}$ in (11) and let $\chi \in C^{\infty}$ be supported in $(9 / 10,11 / 10)$ and equal to 1 near 1 . It is well known that $\left[(1-\chi) h_{\lambda, \gamma}\right] \circ \rho$ is the Fourier transform of an $L^{1}$ function and that the associated maximal operator is of weak type $(1,1)$. The function $\chi h_{\lambda, \gamma}$ can be written as in (14) for $a_{j}=j^{-\gamma}$. Thus $\chi h_{\lambda, \gamma} \in B_{\lambda+1 / 2, s}^{2}$ for $\gamma s>1$. Hence $S_{t}^{\lambda, \gamma} f$ and the associated maximal function are well defined on $L^{p, q}$ if $\lambda=d(1 / 2-1 / p)-1 / 2$ and $\gamma>1-1 / q$.

Finally to show lower bounds we consider the one dimensional inverse Fourier transform $\kappa_{\lambda, \gamma}$ of $\chi h_{\lambda, \gamma}$, i.e.

$$
\begin{aligned}
2 \pi \kappa_{\lambda, \gamma}(r) & =\int \chi(v)(1-v)_{+}^{\lambda}\left(\log \frac{1}{|1-v|}\right)^{-\gamma} e^{i v r} d v \\
& =e^{i r} \int \chi_{0}(t) t_{+}^{\lambda}\left(\log |t|^{-1}\right)^{-\gamma} e^{-i t r} d t
\end{aligned}
$$


here $\chi_{0}$ is supported in $\left(-10^{-1}, 10^{-1}\right)$. By a standard asymptotic expansion ([8], ch. 2.9.), we can estimate the expression that we get by freezing the logarithmic term at $t=r^{-1}$, namely we have for $|r| \gg 1$

$$
\left|\int \chi_{0}(t) t_{+}^{\lambda} e^{-i t r} d t(\log r)^{-\gamma}\right| \gtrsim|r|^{-\lambda-1}(\log |r|)^{-\gamma} .
$$

Straightforward estimation (using integration for the parts where $t \approx r^{-1} 2^{m}$ with $m>0$ ) shows

$$
\left|\int \chi_{0}(t) t_{+}^{\lambda}\left[\left(\log |t|^{-1}\right)^{-\gamma}-(\log r)^{-\gamma}\right] e^{-i t r} d t\right| \lesssim|r|^{-\lambda-1}(\log |r|)^{-\gamma-1} .
$$

The details are left to the reader. In view of the additional logarithmic gain in the last display we obtain for $|r| \gg 1$

$$
\left|\kappa_{\lambda, \gamma}(r)\right| \gtrsim|r|^{-\lambda-1}(\log |r|)^{-\gamma}
$$

From this it is easy to see that the condition

$$
\frac{\kappa_{\lambda, \gamma}}{(1+|r|)^{\frac{d-1}{2}}} \in L^{p^{\prime}, q^{\prime}}\left((1+|r|)^{d-1} d r\right)
$$

implies that either $\lambda>d(1 / 2-1 / p)-1 / 2$ or $\lambda=d(1 / 2-1 / p)-1 / 2$ and $\gamma>1 / q^{\prime}$. Thus Theorem 2.3 and Lemma 2.1 show that the range of $\gamma$ in Theorem 1.1 is optimal.

\section{Preliminaries and basic estimates}

An embedding result. Recall the notation $\mathfrak{A}_{l}=\left\{2^{l} \leq|x|<2^{l+1}\right\}$.

Lemma 3.1. Let $0<a<d, 1<r<\infty$ and let $p=\frac{r d}{d-a}$. Then, for $1<q<\infty$,

$$
\left(\sum_{l \in \mathbb{Z}}\left[\int_{\mathfrak{A}_{l}}|f(x)|^{r}|x|^{-a} d x\right]^{q / r}\right)^{1 / q} \lesssim\|f\|_{L^{p, q}}
$$

and

$$
\sup _{l}\left(\int_{\mathfrak{A}_{l}}|f(x)|^{r}|x|^{-a} d x\right)^{1 / r} \lesssim\|f\|_{L^{p, \infty}}
$$

Proof. Let $f$ be measurable so that $|f(x)| \leq \chi_{E}(x)$ for some set $E$ of finite Lebesgue measure. Let $\varpi_{d}$ be the surface measure of the unit sphere in $\mathbb{R}^{d}$ and let $B(E)$ be the ball centered at the origin, with radius $R(E)=$ $\left(d \varpi_{d}^{-1}|E|\right)^{1 / d}$; then $E$ and $B(E)$ have the same Lebesgue measure and

$$
\int_{E}|x|^{-a} d x \leq \int_{B(E)}|x|^{-a} d x=\frac{d}{d-a}\left(\frac{\varpi_{d}}{d}\right)^{a / d}|E|^{1-\frac{a}{d}} .
$$

This implies the inequality

$$
\left(\int|f(x)|^{r}|x|^{-a} d x\right)^{1 / r} \lesssim\|f\|_{L^{p, 1}}, \quad \frac{1}{p}=\frac{1}{r}-\frac{a}{r d} .
$$


We apply this inequality with two choices $\left(p_{0}, a_{0}\right)$ and $\left(p_{1}, a_{1}\right)$ satisfying $a_{i} / r=d\left(1 / r-1 / p_{i}\right)$ for $i=0,1$ where $a_{0}>0$ is close to 0 and $a_{1}<d$ is close to $d$.

Let $a_{0}<a<a_{1}$ and let $p=\frac{r d}{d-a}$. Then there is $\vartheta \in(0,1)$ so that $\left(\frac{a}{r}, \frac{1}{p}\right)=(1-\vartheta)\left(\frac{a_{0}}{r}, \frac{1}{p_{0}}\right)+\vartheta\left(\frac{a_{1}}{r}, \frac{1}{p_{1}}\right)$. Since $\left[L^{p_{0}, 1}, L^{p_{1}, 1}\right]_{\vartheta, q}=L^{p, q}$ and since $\left[L^{r}\left(|x|^{-a_{0}} d x\right), L^{r}\left(|x|^{-a_{1}} d x\right)\right]_{\vartheta, q}=\dot{\mathcal{K}}_{r}^{-a / r, q}$, by Gilbert's result [11], the assertion follows by interpolation.

Fourier restriction based on traces of Sobolev spaces. In what follows we let $\sigma$ be the surface measure on the $\rho$-unit sphere $\Sigma_{\rho}$. The following result is standard, but we include the proof for completeness.

Lemma 3.2. For $1<b<d$,

$$
\int_{\Sigma_{\rho}}|\widehat{g}(\xi)|^{2} d \sigma(\xi) \lesssim \int|g(x)|^{2}|x|^{b} d x
$$

Proof. We split $g=g_{0}+g_{1}$ where $g_{0}(x)=0$ for $|x|>1$ and $g_{1}(x)=0$ for $|x| \leq 1$. Then

$$
\begin{aligned}
& \left(\int_{\Sigma_{\rho}}\left|\widehat{g}_{0}(\xi)\right|^{2} d \sigma(\xi)\right)^{1 / 2} \lesssim\left\|\widehat{g}_{0}\right\|_{\infty} \lesssim\left\|g_{0}\right\|_{1} \\
& \lesssim\left(\int\left|g_{0}(x)\right|^{2}|x|^{b} d x\right)^{1 / 2}\left(\int_{|x| \leq 1}|x|^{-b} d x\right)^{1 / 2} \lesssim\left(\int|g(x)|^{2}|x|^{b} d x\right)^{1 / 2}
\end{aligned}
$$

where we have used $b<d$.

Now the trace theorem says that for $b>1$ the restriction to $\Sigma_{\rho}$ of functions in the Sobolev space $\mathcal{L}_{b / 2}^{2}\left(\mathbb{R}^{d}\right)$ belongs to $\mathcal{L}_{(b-1) / 2}^{2}\left(\Sigma_{\rho}\right)$, so it is in $L^{2}\left(\Sigma_{\rho}\right)$. We apply the corresponding inequality to $\widehat{g}_{1}$ and combine it with Plancherel's theorem to see that

$$
\left(\int_{\Sigma_{\rho}}\left|\widehat{g}_{1}(\xi)\right|^{2} d \sigma(\xi)\right)^{1 / 2} \lesssim\left(\int\left|g_{1}(x)\right|^{2}\left(1+|x|^{2}\right)^{b / 2} d x\right)^{1 / 2}
$$

In view of the support of $g_{1}$ we may replace the weight $\left(1+|x|^{2}\right)^{b / 2}$ with $|x|^{b}$ and then $g_{1}$ with $g$. Finally, combine the estimates for $\widehat{g}_{0}$ and $\widehat{g}_{1}$.

Estimates for maximal functions. We use Lemma 3.2 to bound a maximal operator associated with $h \circ \rho$.

Proposition 3.3. Let $1<b<d$. Let $\rho \in C^{\infty}\left(\mathbb{R}^{d} \backslash\{0\}\right.$, homogeneous of degree $\beta>0$ and positive on $\mathbb{R}^{d} \backslash\{0\}$. Suppose that

$$
\left(\int_{0}^{\infty}|h(s)|^{2} s^{\frac{b}{\beta}-1} d s\right)^{1 / 2} \leq A
$$

Then

$$
\left(\left.\int\left|\sup _{1<t<2}\right| \mathcal{F}^{-1}[h(\rho(\cdot) / t) \widehat{f}]\right|^{2} \frac{d x}{|x|^{b}}\right)^{1 / 2} \lesssim A\|f\|_{2}
$$


Proof. This follows from the dual inequality

$$
\left\|\int_{1}^{2} \mathcal{F}^{-1}\left[h \circ(\rho / t) \widehat{f_{t}}\right] d t\right\|_{L^{2}} \lesssim A\left\|\int_{1}^{2}\left|f_{t}\right| d t\right\|_{L^{2}\left(|x|^{b} d x\right)} .
$$

Using Plancherel's theorem and generalized polar coordinates $\xi=\rho \xi^{\prime}$, $\rho>0, \xi^{\prime} \in \Sigma_{\rho}$, we can write the left hand side of (16) as

$$
\left(\int \rho^{\frac{d}{\beta}-1} \int_{\Sigma_{\rho}}\left|\int h(\rho / t) \widehat{f}_{t}\left(\rho^{1 / \beta} \xi^{\prime}\right) d t\right|^{2} \frac{d \sigma\left(\xi^{\prime}\right)}{\beta\left|\nabla \rho\left(\xi^{\prime}\right)\right|} d \rho\right)^{1 / 2} .
$$

Since $|\nabla \rho|$ is bounded below we can drop this term and use Lemma 3.2 to get

$$
\begin{aligned}
\| \int_{1}^{2} \mathcal{F}^{-1}[h \circ & \left.(\rho / t) \widehat{f_{t}}\right] d t \|_{L^{2}} \\
& \lesssim\left(\int \rho^{\frac{d}{\beta}-1} \int|x|^{b}\left|\int h(\rho / t) \rho^{-d / \beta} f_{t}\left(\rho^{-1 / \beta} x\right) d t\right|^{2} d x d \rho\right)^{1 / 2} .
\end{aligned}
$$

We change variables and the last expression becomes

$$
\begin{aligned}
& \left(\int|x|^{b} \int \rho^{\frac{b}{\beta}-1}\left|\int h(\rho / t) f_{t}(x) d t\right|^{2} d x d \rho\right)^{1 / 2} \\
& \leq\left(\int|x|^{b}\left[\int_{1}^{2}\left(\int|h(\rho / t)|^{2} \rho^{\frac{b}{\beta}-1} d \rho\right)^{1 / 2}\left|f_{t}(x)\right| d t\right]^{2} d x\right)^{1 / 2} \\
& \leq\left(\int \rho^{\frac{b}{\beta}-1}|h(\rho)|^{2} d \rho\right)^{1 / 2}\left(\int\left[\int_{1}^{2} t^{\frac{b}{\beta}}\left|f_{t}(x)\right| d t\right]^{2}|x|^{b} d x\right)^{1 / 2} .
\end{aligned}
$$

The assertion follows.

In the proof of Theorem 1.5 we shall use

Proposition 3.4. Let $1<b<d$ and $\rho$ as in Proposition 3.3. Let $\eta \in C^{\infty}$ be supported in $\left(\frac{1}{8}, 8\right)$. For any real $\tau$ define $\mathcal{A}_{\tau}$ by

$$
\widehat{\mathcal{A}_{\tau} f}(\xi)=\eta(\rho(\xi)) e^{-i \rho(\xi) \tau} \widehat{f}(\xi) .
$$

Then

$$
\int_{-\infty}^{\infty}\left\|\mathcal{A}_{\tau} f\right\|_{L^{2}\left(|x|^{-b}\right)}^{2} d \tau \lesssim\|f\|_{2}^{2}
$$

Proof. The inequality is equivalent with

$$
\left\|\int \mathcal{A}_{\tau}[g(\tau, \cdot)] d \tau\right\|_{2} \lesssim\left(\int\|g(\tau, \cdot)\|_{L^{2}\left(|x|^{b}\right)}^{2} d \tau\right)^{1 / 2} .
$$

Using Plancherel's theorem and generalized polar coordinates we see that the square of the left hand side is bounded by

$$
\begin{aligned}
& \int|\eta(\rho)|^{2} \int_{\Sigma_{\rho}}\left|\int e^{i \tau \rho} \widehat{g}\left(\tau, \rho^{1 / \beta} \xi^{\prime}\right) d \tau\right|^{2} \frac{d \sigma\left(\xi^{\prime}\right)}{\left|\nabla \rho\left(\xi^{\prime}\right)\right|} \rho^{\frac{d}{\beta}-1} d \rho \\
& \lesssim \int|\eta(\rho)|^{2} \int\left|\int e^{i \tau \rho} \rho^{-d / \beta} g\left(\tau, \rho^{-1 / \beta} x\right) d \tau\right|^{2}|x|^{b} d x \rho^{\frac{d}{\beta}-1} d \rho
\end{aligned}
$$


where we have used Lemma 3.2. By a change of variables the last expression is

$$
\begin{aligned}
& \int|\eta(\rho)|^{2} \rho^{\frac{b}{\beta}-1} \int\left|\int e^{i \tau \rho} g(\tau, x) d \tau\right|^{2}|x|^{b} d x d \rho \\
& \lesssim\left\|\left(\int\left|\int e^{i \tau \rho} g(\tau, \cdot) d \tau\right|^{2} d \rho\right)^{1 / 2}\right\|_{L^{2}\left(|x|^{b}\right) d x}^{2} \\
& \lesssim\left\|\left(\int|g(\tau, \cdot)|^{2} d \tau\right)^{1 / 2}\right\|_{L^{2}\left(|x|^{b}\right) d x}^{2}
\end{aligned}
$$

where the last inequality holds by Plancherel's theorem in the first variable. Now (18) follows.

Littlewood-Paley inequalities. Let $\mathfrak{A}$ be a compact subset of $\mathbb{R}^{d} \backslash\{0\}$ and let $\left\{\zeta_{k}\right\}$ be a bounded family in $C^{\infty}$, so that all $\zeta_{k}$ are supported in $\mathfrak{A}$. Define operators $P_{k}$ by $\widehat{P_{k} f}(\xi)=\zeta_{k}\left(2^{-k} \xi\right) \widehat{f}$.

Lemma 3.5. Let $-d / 2<\gamma<d / 2$. Then for all $f \in \dot{\mathcal{K}}_{2}^{\gamma, q}$,

$$
\left\|\left(\sum_{k}\left|P_{k} f\right|^{2}\right)^{1 / 2}\right\|_{\dot{\mathcal{K}}_{2}^{\gamma, q}} \leq C(\mathfrak{A}, \gamma)\|f\|_{\dot{\mathcal{K}}_{2}^{\gamma, q}}
$$

and for all $\ell^{2}$ valued functions $F=\left\{f_{k}\right\}_{k \in \mathbb{Z}} \in \dot{\mathcal{K}}_{2}^{\gamma, q}\left(\ell^{2}\right)$

$$
\left\|\sum_{k} P_{k} f_{k}\right\|_{\dot{\mathcal{K}}_{2}^{\gamma, q}} \leq C(\mathfrak{A}, \gamma)\left\|\left(\sum_{k}\left|f_{k}\right|^{2}\right)^{1 / 2}\right\|_{\dot{\mathcal{K}}_{2}^{\gamma, q}} \text {. }
$$

Proof. Since $|x|^{-2 \gamma}$ is an $A_{2}$ weight for $-d / 2<\gamma<d / 2$ the LittlewoodPaley inequalities hold for the weighted $L^{2}\left(|x|^{2 \gamma} d x\right)$ spaces, i.e. for $\dot{\mathcal{K}}_{2}^{\gamma, 2}$. The case for general $q$ follows by real interpolation.

Reduction to the case of homogeneity $\beta=1$. In the proof of our main theorems we may assume $\beta=1$. This follows from writing $h(\rho(\xi))=$ $h_{\beta}\left(\rho(\xi)^{1 / \beta}\right)$ with $h_{\beta}(s)=h\left(s^{\beta}\right)$ and the following lemma.

Lemma 3.6. Let $\alpha>0, \beta>0$. Then for all $h \in B_{\alpha, q}^{2}(\mathbb{R})$ supported in a compact interval $J \subset(0, \infty)$

$$
\left\|h\left((\cdot)^{\beta}\right)\right\|_{B_{\alpha, q}^{2}} \leq C_{\beta, J}\|h\|_{B_{\alpha, q}^{2}} .
$$

Proof. Let $J=[a, b]$ and $J_{\beta}=\left[a^{1 / \beta}, b^{1 / \beta}\right]$. Let $\chi \in C_{0}^{\infty}$ be compactly supported in $(0, \infty)$ and equal to 1 on $J_{\beta}$. Then we have $\left\|\chi(\cdot) h\left((\cdot)^{\beta}\right)\right\|_{\mathcal{L}_{k}^{2}} \leq$ $C(\chi, \beta)\|h\|_{B_{\alpha, q}^{2}}$ for $k=0,1,2, \ldots$, by straightforward computation. The corresponding inequality for Besov spaces $B_{\alpha, q}^{2}$ for all $\alpha>0$ follows by real interpolation.

\section{Proof of Theorem 1.2}

In what follows we shall assume $\beta=1$, as we may by the last subsection in 93 . 
The basic decompositions. Let $h \in B_{\alpha, q^{\prime}}^{2}(\mathbb{R})$ be supported in $(1 / 2,2)$ and let $\eta$ be a $C^{\infty}$ function, supported on $(1 / 8,8)$ such that $\eta(s)=1$ on $(1 / 4,4)$. Define $L_{k}$ by $\widehat{L_{k} f}(\xi)=\eta\left(2^{-k} \rho(\xi)\right) \widehat{f}(\xi)$. Then

$$
\begin{aligned}
\sup _{s>0}\left|\mathcal{F}^{-1}\left[h\left(\frac{\rho}{s}\right) \widehat{f}\right]\right| & =\sup _{k} \sup _{1 \leq t \leq 2}\left|\mathcal{F}^{-1}\left[h\left(\frac{\rho}{2^{k} t}\right) \widehat{L_{k} f}\right]\right| \\
& =\left(\sum_{k} \sup _{1 \leq t \leq 2}\left|\mathcal{F}^{-1}\left[h\left(\frac{\rho}{2^{k}}\right) \widehat{L_{k} f}\right]\right|^{2}\right)^{1 / 2} .
\end{aligned}
$$

Let $\zeta_{0} \in C^{\infty}(\mathbb{R})$ be supported in $(-1,1)$ such that $\zeta_{0}(r)=1$ for $|r| \leq 1 / 2$ and let, for $j \geq 1, \zeta_{j}(r)=\zeta_{0}\left(2^{-j} r\right)-\zeta_{0}\left(2^{-j+1} r\right)$. Define

$$
V_{j} h(\rho)=(2 \pi)^{-1} \int \zeta_{j}(r) \widehat{h}(r) e^{i r \rho} d r
$$

so that $\widehat{V_{j} h}$ is supported in $I_{j}=\left[2^{j-2}, 2^{j}\right] \cup\left[-2^{j},-2^{j-2}\right]$ for $j=1,2, \ldots$, and $\sum_{j=0}^{\infty} V_{j} h=h$. Define

$$
T_{s}^{j, k}[h, f]=\mathcal{F}^{-1}\left[\eta\left(\rho\left(2^{-k} \cdot\right)\right) V_{j} h\left(2^{-k} \frac{\rho(\cdot)}{s}\right) \widehat{f}\right] .
$$

We first note that for large $j$ the Fourier transform of $\eta(\rho(\cdot)) V_{j} h(\rho(\cdot))$ is concentrated on an annulus of width $\approx 2^{j}$. By assumption, there is $c_{0} \in \mathbb{N}$ so that

$$
2^{-c_{0}+2} \leq|\nabla \rho(\xi)| \leq 2^{c_{0}-2}, \text { for } 1 / 8 \leq \rho(\xi) \leq 8 .
$$

Lemma 4.1. Let $K_{j, s}$ be defined by $\widehat{K}_{j, s}(\xi)=\eta(\rho(\xi)) V_{j} h(\rho(\xi) / s)$. Then for $j=0,1,2, \ldots$, and $1 / 2 \leq s \leq 2$,

$$
\begin{aligned}
& \left|K_{j, s}(x)\right| \leq C_{N}\|h\|_{\infty} 2^{-j N}|x|^{-N}, \text { for }|x| \geq 2^{j+c_{0}}, \\
& \left|K_{j, s}(x)\right| \leq C_{N}\|h\|_{\infty} 2^{-j N}, \text { for }|x| \leq 2^{-j-c_{0}}
\end{aligned}
$$

Proof. First observe that $\left\|\widehat{V_{j} h}\right\|_{1} \lesssim 2^{j}\|h\|_{\infty}$. We write

$$
K_{j, s}(x)=\frac{1}{(2 \pi)^{d+1}} \int \widehat{V_{j} h}(\tau) \int \eta(\rho(\xi)) e^{i(\tau \rho(\xi)+\langle x, \xi\rangle)} d \xi d \tau
$$

and observe that, for $\xi \in \operatorname{supp}(\eta \circ \rho)$,

$$
\left|\nabla_{\xi}(\tau \rho(\xi)+\langle x, \xi\rangle)\right| \geq \max \left\{|x|-2^{j+c_{0}-1}, 2^{j-c_{0}}-|x|\right\}
$$

Multiple integration by parts in $\xi$ yields the asserted estimate.

Let $\chi_{l}$ be the indicator function of the annulus $\mathfrak{A}_{l}$. The above lemma suggests to estimate the maximal square-function (19) by

$$
\left(\sum_{k} \mathcal{M}_{1}^{k}\left[L_{k} f, h\right]^{2}\right)^{1 / 2}+\left(\sum_{k} \mathcal{M}_{2}^{k}\left[L_{k} f, h\right]^{2}\right)^{1 / 2}
$$


where

$$
\begin{aligned}
& \mathcal{M}_{1}^{k}[f, h]=\sup _{1 \leq t \leq 2}\left|\sum_{j=0}^{\infty} T_{t}^{j, k}\left[h, \sum_{n \geq-c_{0}} \chi_{-k+j-n} f\right]\right|, \\
& \mathcal{M}_{2}^{k}[f, h]=\sup _{1 \leq t \leq 2}\left|\sum_{j=0}^{\infty} T_{t}^{j, k}\left[h, \sum_{l>j+c_{0}} \chi_{-k+l} f\right]\right| .
\end{aligned}
$$

For the second term we can reduce to straightforward $L^{2}$ estimates for which the weight plays little role. The first term requires the condition $h \in B_{\alpha, s}^{2}$ but we get a bound even for $\sum_{k} \mathcal{M}_{1}^{k}\left[f_{k}, h\right]$ in place of the square-function.

Proposition 4.2. Let $1 / 2<\alpha<d / 2, s=\frac{q}{q-1}$ and let $h \in B_{\alpha, s}^{2}$ be supported in $\left(\frac{1}{2}, 2\right)$.

(i) If $2 \leq q \leq \infty$ then

$$
\left\|\sum_{k} \mathcal{M}_{1}^{k}\left[f_{k}, h\right]\right\|_{\dot{\mathcal{K}}_{2}^{-\alpha, q}} \lesssim\|h\|_{B_{\alpha, s}^{2}}\left\|\left(\sum_{k}\left|f_{k}\right|^{q}\right)^{1 / q}\right\|_{\dot{\mathcal{K}}_{2}^{-\alpha, q}} .
$$

(ii) If $1 \leq q \leq 2$ then

$$
\left\|\sum_{k} \mathcal{M}_{1}^{k}\left[f_{k}, h\right]\right\|_{\dot{\mathcal{K}}_{2}^{-\alpha, 2}} \lesssim\|h\|_{B_{\alpha, s}^{2}}\left\|\left(\sum_{k}\left|f_{k}\right|^{2}\right)^{1 / 2}\right\|_{\dot{\mathcal{K}}_{2}^{-\alpha, q}} .
$$

Proposition 4.3. Let $1 / 2<\alpha<d / 2, \gamma>1 / 2$ and let $h \in B_{\gamma, 1}^{2}$ be supported in $\left(\frac{1}{2}, 2\right)$. Then

$$
\left\|\left(\sum_{k} \mathcal{M}_{2}^{k}\left[f_{k}, h\right]^{2}\right)^{1 / 2}\right\|_{\dot{\mathcal{K}}_{2}^{-\alpha, q}} \lesssim\|h\|_{B_{\gamma, 1}^{2}}\left\|\left(\sum_{k}\left|f_{k}\right|^{2}\right)^{1 / 2}\right\|_{\dot{\mathcal{K}}_{2}^{-\alpha, q}} .
$$

We prove these propositions in the following two subsections.

Estimates for $\mathcal{M}_{1}$. We localize both on the function and the operator sides. The basic estimate is

Lemma 4.4. Let $1<b<d$ and $1 / 2<\alpha<d / 2$. Let $V_{j}$ be as in (20) and

$$
\Lambda_{b}^{j}(h)=\left(\int\left|V_{j} h(\rho)\right|^{2} \rho^{b-1} d \rho\right)^{1 / 2} .
$$

For $k \in \mathbb{Z}, m \in \mathbb{Z}$, and $n \geq-c_{0}$,

$$
\begin{aligned}
\| \chi_{m-k} \sup _{1 \leq t \leq 2}\left|\sum_{j=0}^{\infty} T_{t}^{j, k}\left[h, g \chi_{-k+j-n}\right]\right| & \|_{L^{2}\left(|x|^{-2 \alpha} d x\right)} \\
& \lesssim 2^{-n \alpha} 2^{m\left(\frac{b}{2}-\alpha\right)} \sum_{j \geq 0} 2^{j \alpha} \Lambda_{b}^{j}(h)\left\|g \chi_{j-n-k}\right\|_{L^{2}\left(|x|^{-2 \alpha} d x\right)}
\end{aligned}
$$


Proof. On the support of $\chi_{m-k}$ we have $|x|^{-2 \alpha} \approx 2^{(k-m)(2 \alpha-b)}|x|^{-b}$ and by Minkowski's inequality

$$
\begin{aligned}
& \left\|\chi_{m-k} \sup _{1 \leq t \leq 2}\left|\sum_{j=0}^{\infty} T_{t}^{j, k}\left[h, g \chi_{-k+j-n}\right]\right|\right\|_{L^{2}\left(|x|^{-2 \alpha} d x\right)} \\
& \lesssim 2^{(k-m) \frac{2 \alpha-b}{2}} \sum_{j=0}^{\infty}\left\|\sup _{1 \leq t \leq 2}\left|T_{t}^{j, k}\left[h, g \chi_{-k+j-n}\right]\right|\right\|_{L^{2}\left(|x|^{-b} d x\right)} .
\end{aligned}
$$

Note that $T_{t}^{j, k}[h, g](x)=T_{t}^{j, 0}\left[h, g\left(2^{-k} \cdot\right)\right]\left(2^{k} x\right)$ and hence

$$
\begin{aligned}
& \left\|\sup _{1 \leq t \leq 2}\left|T_{t}^{j, k}\left[h, g \chi_{-k+j-n}\right]\right|\right\|_{L^{2}\left(|x|^{-b} d x\right)} \\
& =2^{-\frac{d-b}{2}}\left\|\sup _{1 \leq t \leq 2}\left|T_{t}^{j, 0}\left[h, g\left(2^{-k \cdot}\right) \chi_{j-n}\right]\right|\right\|_{L^{2}\left(|x|^{-b} d x\right)} \\
& \lesssim 2^{-k \frac{d-b}{2}} \Lambda_{b}^{j}(h)\left\|g\left(2^{-k} \cdot\right) \chi_{j-n}\right\|_{2}
\end{aligned}
$$

where we have applied Proposition 3.3. The last displayed quantity is equal to

$$
2^{k b / 2} \Lambda_{b}^{j}(h)\left\|g \chi_{-k+j-n}\right\|_{2} \lesssim 2^{k \frac{b-2 \alpha}{2}} 2^{(j-n) \alpha} \Lambda_{b}^{j}(h)\left\|g \chi_{-k+j-n}\right\|_{L^{2}\left(|y|^{-2 \alpha} d y\right)}
$$

and the asserted inequality follows if we use this in (26).

Lemma 4.5. Let $h \in L^{2}(\mathbb{R})$ be supported in $\left(\frac{1}{2}, 2\right)$ and $\Lambda_{b}^{j}(h)$ be as in (25). Then for $b>0, \alpha>0$

$$
\left(\sum_{j=0}^{\infty}\left[2^{j \alpha} \Lambda_{b}^{j}(h)\right]^{s}\right)^{1 / s} \lesssim\|h\|_{B_{\alpha, s}^{2}} .
$$

Proof. Let

$$
\widetilde{\Lambda}_{b}^{j}(h)=\int_{1 / 4}^{4}\left|V_{j} h(\rho)\right|^{2} \rho^{b-1} d \rho .
$$

Then the corresponding estimate with $\Lambda_{b}^{j}$ replaced by $\widetilde{\Lambda}_{b}^{j}$ is obvious from the definition of Besov spaces. To estimate the corresponding contribution over $\mathbb{R} \backslash[1 / 4,4]$ write

$$
2 \pi V_{j} h(\rho)=\int h(u) \int \chi_{j}(r) e^{i(\rho-u) r} d r d u
$$

By integration by parts, the inner integral is $\leq C_{N} 2^{-j N}|\rho-u|^{-N-1}$ and since $h$ is supported in $\left[\frac{1}{2}, 2\right]$ we see that $\left|V_{j} h(\rho)\right| \lesssim\|h\|_{1} 2^{-j N}$ for $\rho \leq 1 / 4$ and $\left|V_{j} h(\rho)\right| \lesssim\|h\|_{1} 2^{-j N} \rho^{-N}$ for $|\rho| \geq 4$. A straightforward estimate gives the assertion.

Proof of Proposition 4.2 for $q \geq 2$. Let

$$
\mathcal{M}_{m, n}^{k} f=\chi_{m-k} \sup _{1 \leq t \leq 2}\left|\sum_{j} T_{t}^{j, k}\left[h, \chi_{-k+j-n} f\right]\right| .
$$


Then, by Minkowski's inequality,

$$
\left\|\sum_{k} \mathcal{M}_{1}^{k}[f, h]\right\|_{\dot{\mathcal{K}}_{2}^{-\alpha, q}} \leq \sum_{m=-\infty}^{\infty} \sum_{n=-c_{0}}^{\infty}\left\|\sum_{k} \mathcal{M}_{m, n}^{k}[f, h]\right\|_{\dot{\mathcal{K}}_{2}^{-\alpha, q}} .
$$

We use the estimate

$$
\left\|\sum_{k} \chi_{m-k} g_{k}\right\|_{\dot{\mathcal{K}}_{2}^{-\alpha, q}} \lesssim\left(\sum_{k}\left\|\chi_{m-k} g_{k}\right\|_{L^{2}\left(|x|^{-2 \alpha}\right)}^{q}\right)^{1 / q}
$$

which holds for all $q \geq 1$ and all $\alpha \in \mathbb{R}$ and is obvious from the definition of the $\dot{\mathcal{K}}_{2}^{-\alpha, q}$ spaces. We now choose $b_{1}, b_{2}$ such that $1<b_{1}<2 \alpha$ and $2 \alpha<b_{2}<d$. From (28), (29) and Lemma 4.4 we get

$$
\begin{aligned}
& \left\|\sum_{k} \mathcal{M}_{1}^{k}\left[f_{k}, h\right]\right\|_{\mathcal{K}_{2}^{-\alpha, q}} \lesssim \sum_{n \geq-c_{0}} 2^{-n \alpha} \times \\
& \quad\left[\sum_{m \geq 0} 2^{-m \frac{2 \alpha-b_{1}}{2}}\left(\sum_{k}\left|\mathcal{E}_{b_{1}, n, k}\right|^{q}\right)^{1 / q}+\sum_{m<0} 2^{m \frac{b_{2}-2 \alpha}{2}}\left(\sum_{k}\left|\mathcal{E}_{b_{2}, n, k}\right|^{q}\right)^{1 / q}\right]
\end{aligned}
$$

where

$$
\mathcal{E}_{b, n, k}=\sum_{j} 2^{j \alpha} \Lambda_{b}^{j} h\left\|f_{k} \chi_{j-n-k}\right\|_{L^{2}\left(|y|^{-2 \alpha} d y\right)} .
$$

Now the proof is concluded by Lemma 4.5 and estimating (with $b=b_{1}$ or $\left.b=b_{2}\right)$

$$
\begin{aligned}
& \left(\sum_{k}\left|\mathcal{E}_{b, n, k}\right|^{q}\right)^{1 / q} \\
& \lesssim\left(\sum_{k}\left(\sum_{j}\left[2^{j \alpha} \Lambda_{b}^{j}(h)\right]^{s}\right)^{q / s} \sum_{j}\left\|f_{k} \chi_{j-n-k}\right\|_{L^{2}\left(|y|^{-2 \alpha} d y\right)}^{q}\right)^{1 / q} \\
& =\left(\sum_{j}\left[2^{j \alpha} \Lambda_{b}^{j}(h)\right]^{s}\right)^{1 / s}\left(\sum_{l} \sum_{k}\left\|f_{k} \chi_{l}\right\|_{L^{2}\left(|y|^{-2 \alpha}\right)}^{q}\right)^{1 / q} \\
& \lesssim\|h\|_{B_{\alpha, s}^{2}}\left\|\left(\sum_{k}\left|f_{k}\right|^{q}\right)^{1 / q}\right\|_{\dot{\mathcal{K}}_{2}^{-\alpha, q}} .
\end{aligned}
$$

Proof of Proposition 4.2 for $q \leq 2$. Again we work with (27), use (28), (29) for $q=2$ and Lemma 4.4 to get

$$
\begin{aligned}
& \left\|\sum_{k} \mathcal{M}_{1}^{k}\left[f_{k}, h\right]\right\|_{\dot{\mathcal{K}}_{2}^{-\alpha, 2}} \lesssim \sum_{n \geq-c_{0}} 2^{-n \alpha} \times \\
& \quad\left[\sum_{m \geq 0} 2^{-m \frac{2 \alpha-b_{1}}{2}}\left(\sum_{k}\left|\mathcal{E}_{b_{1}, n, k}\right|^{2}\right)^{1 / 2}+\sum_{m<0} 2^{m \frac{b_{2}-2 \alpha}{2}}\left(\sum_{k}\left|\mathcal{E}_{b_{2}, n, k}\right|^{2}\right)^{1 / 2}\right],
\end{aligned}
$$


with $\mathcal{E}_{b, n, k}$ as in (30), and $b_{1} \in(1,2 \alpha)$ and $b_{2} \in(2 \alpha, d)$. Again we apply Hölder's inequality and get, for fixed $m, n$,

$$
\begin{aligned}
& \left(\sum_{k}\left|\mathcal{E}_{b, n, k}\right|^{2}\right)^{1 / 2} \\
& \leq\left(\sum_{k}\left(\sum_{j}\left[2^{j \alpha} \Lambda_{b}^{j}(h)\right]^{s}\right)^{2 / s}\left(\sum_{j}\left\|f_{k} \chi_{j-n-k}\right\|_{L^{2}\left(|y|^{-2 \alpha} d y\right)}^{q}\right)^{2 / q}\right)^{1 / 2} \\
& \leq\left(\sum_{j}\left[2^{j \alpha} \Lambda_{b}^{j}(h)\right]^{s}\right)^{1 / s}\left(\sum_{k}\left[\sum_{l}\left\|f_{k} \chi_{l}\right\|_{L^{2}\left(|y|^{-2 \alpha} d y\right)}^{q}\right]^{2 / q}\right)^{1 / 2}
\end{aligned}
$$

and since now $q \leq 2$ we may use Minkowski's inequality and estimate this by

$$
\begin{aligned}
& \|h\|_{B_{\alpha, s}^{2}}\left(\sum_{l}\left\|\chi_{l}\left(\sum_{k}\left|f_{k}\right|^{2}\right)^{1 / 2}\right\|_{L^{2}\left(|y|^{-2 \alpha} d y\right)}^{q}\right)^{1 / q} \\
& \lesssim\|h\|_{B_{\alpha, s}^{2}}\left\|\left(\sum_{k}\left|f_{k}\right|^{2}\right)^{1 / 2}\right\|_{\dot{\mathcal{K}}_{2}^{-\alpha, q}} .
\end{aligned}
$$

Estimates for $\mathcal{M}_{2}$. For every $l$ let $\widetilde{\chi}_{l}=\sum_{i=-2-c_{0}}^{2+c_{0}} \chi_{l}$. We estimate

$$
\mathcal{M}_{2}^{k}[f, h] \leq \sum_{j \geq 1}\left(\widetilde{\mathcal{M}}_{2, j}^{k}[f, h]+\mathcal{N}_{j}^{k}[f, h]\right)
$$

where

$$
\begin{aligned}
\widetilde{\mathcal{M}}_{2, j}^{k}[f, h] & =\sum_{l>j+c_{0}} \sup _{1 \leq t \leq 2}\left|\widetilde{\chi}_{-k+l} T_{2^{k} t}^{j}\left[h, \chi_{-k+l} f\right]\right| \\
\mathcal{N}_{j}^{k}[f, h] & =\sum_{l>j+c_{0}} \sup _{1 \leq t \leq 2}\left|\left(1-\tilde{\chi}_{-k+l}\right) \sum_{j} T_{2^{k} t}^{j}\left[h, \chi_{-k+l} f\right]\right|
\end{aligned}
$$

For the estimation of $\left(\sum_{k} \widetilde{\mathcal{M}}_{2, j}^{k}[f, h]^{2}\right)^{1 / 2}$ we use a standard imbedding estimate.

Lemma 4.6. For $\gamma>1 / 2$

$$
\left\|\sup _{1 \leq t \leq 2}\left|T_{2^{k} t}^{j}[h, f]\right|\right\|_{2} \leq 2^{j\left(\frac{1}{2}-\gamma\right)}\|h\|_{B_{\gamma, 1}^{2}}\|f\|_{L^{2}\left(\mathbb{R}^{d}\right)} .
$$

Proof. By scaling it suffices to prove this for $k=0$. For $g$ supported in $(1 / 2,2)$ and $I=[1 / 2,2]$,

$$
\left\|\sup _{t \in I}\left|\mathcal{F}^{-1}\left[g\left(\frac{\rho(\cdot)}{t}\right) \widehat{f}\right]\right|\right\|_{2} \leq\left[\|g\|_{2}+\|g\|_{2}^{1 / 2}\left\|g^{\prime}\right\|_{2}^{1 / 2}\right]\|f\|_{2}
$$

as one can see by applying the fundamental theorem of calculus to $g^{2}$. Since $\left\|V_{j} h\right\|_{2}+2^{-j}\left\|\left(V_{j} h\right)^{\prime}\right\|_{2} \leq 2^{-j \gamma}\|h\|_{B_{\gamma, 1}^{2}}$, the assertion follows. 
Proof of Proposition 4.3. From Lemma 4.6 we get, considering the supports of the functions $\chi_{-k+l}$ and $\tilde{\chi}_{-k+l}$,

$$
\begin{aligned}
& \left\|\left(\sum_{k} \widetilde{\mathcal{M}}_{2, j}^{k}\left[f_{k}, h\right]^{2}\right)^{1 / 2}\right\|_{L^{2}\left(|x|^{-2 \alpha} d x\right)} \\
& \lesssim\left(\sum_{l>c_{0}+j} \sum_{k} 2^{2 \alpha(k-l)}\left\|\sup _{1 \leq t \leq 2}\left|T_{t}^{j, k}\left[h, \chi_{-k+l} f_{k}\right]\right|\right\|_{2}^{2}\right)^{1 / 2} \\
& \lesssim 2^{j\left(\frac{1}{2}-\gamma\right)}\|h\|_{B_{\gamma, 1}^{2}}\left(\sum_{l} \sum_{k} 2^{2 \alpha(k-l)}\left\|f_{k} \chi_{-k+l}\right\|_{2}^{2}\right)^{1 / 2} \\
& \lesssim 2^{j\left(\frac{1}{2}-\gamma\right)}\|h\|_{B_{\gamma, 1}^{2}}\left\|\left(\sum_{k}\left|f_{k}\right|^{2}\right)^{1 / 2}\right\| \|_{L^{2}\left(|x|^{-2 \alpha} d x\right)} .
\end{aligned}
$$

From Lemma 4.1 we get

$$
\mathcal{N}_{j}^{k}[f, h](x) \lesssim 2^{-j N}\|h\|_{\infty} \int \frac{2^{k d}}{\left(1+2^{k}|x-y|\right)^{N}}|f(y)| d y,
$$

and since $|x|^{-2 \alpha}$ is an $A_{2}$ weight for $|\alpha|<d / 2$ the estimate

$$
\left\|\left(\sum_{k} \mathcal{N}_{j}^{k}\left[f_{k}, h\right]^{2}\right)^{1 / 2}\right\|_{L^{2}\left(|x|^{-2 \alpha} d x\right)} \lesssim 2^{-j N}\|h\|_{\infty}\left\|\left(\sum_{k}\left|f_{k}\right|^{2}\right)^{1 / 2}\right\| \|_{L^{2}\left(|x|^{-2 \alpha} d x\right)}
$$

follows from standard results (see ch. IV in [10]). Proposition 4.3 follows by combining the estimates for $\left\{\widetilde{\mathcal{M}}_{2, j}^{k}\right\}$ and $\left\{\mathcal{N}_{j}^{k}\right\}$.

Proof of Theorem 1.2. By (19), the decomposition described in 84 and Propositions 4.2 and 4.3 we have the estimates

$$
\left\|\sup _{t>0}\left|\mathcal{F}^{-1}\left[h\left(\frac{\rho(\cdot)}{t}\right) \widehat{f}\right]\right|\right\|_{\dot{\mathcal{K}}_{2}^{-\alpha, \sigma}} \lesssim\left\|\left(\sum_{k}\left|P_{k} f\right|^{2}\right)^{1 / 2}\right\|_{\dot{\mathcal{K}}_{2}^{-\alpha, q}}, \sigma=\max \{q, 2\},
$$

where the $P_{k}$ are suitable Littlewood-Paley operators as used in Lemma 3.5, satisfying $L_{k} P_{k}=L_{k}$. We conclude by an application of the first inequality in Lemma 3.5

\section{Conclusion of Other PROOFS}

Proof of Theorem 1.4. $(i)$ and $(i i)$ are equivalent by duality.

Assume (ii) holds. Define $T_{t}$ by $\widehat{T_{t} f}(\xi)=m(t \rho(\xi)) \widehat{f}(\xi)$. Then $T_{t}$ is bounded on $L^{2}\left(|x|^{2 \alpha}\right)=\dot{\mathcal{K}}_{2}^{\alpha, 2}$, with operator norm independent of $t$, by the homogeneity of $\rho$ and scale invariance. Test $T_{t}$ on Schwartz functions of the form $\varphi \circ \rho$ to derive the condition (iii).

Finally if $($ iii $)$ holds, let $\chi \in C^{\infty}(\mathbb{R})$ be supported in $(1 / 2,2)$ so that $\sum_{k \in \mathbb{Z}}\left[\chi\left(2^{-k} s\right)\right]^{3}=1$. Define $P_{k}$ and $T_{k}$ by $\widehat{P_{k} f}(\xi)=\chi\left(2^{-k} \rho(\xi)\right) \widehat{f}(\xi)$ and $\widehat{T_{k} f}(\xi)=\chi\left(2^{-k} \rho(\xi)\right) m(\rho(\xi)) \widehat{f}(\xi)$. It follows from Propositions 4.2 and 4.3 
that $P_{k} T_{k} P_{k}$ is bounded on $\dot{\mathcal{K}}_{2}^{-\alpha, 2}$ with operator norm $\lesssim\left\|\chi m\left(2^{k} \cdot\right)\right\|_{\mathcal{L}_{\alpha}^{2}}$. Hence by interchanging sums and integrals

$$
\left\|\left(\sum_{k}\left|P_{k} T_{k} P_{k} f\right|^{2}\right)^{1 / 2}\right\|_{\dot{\mathcal{K}}_{2}^{-\alpha, 2}} \lesssim \sup _{k}\left\|\chi m\left(2^{k} \cdot\right)\right\|_{\mathcal{L}_{\alpha}^{2}}\left\|\left(\sum_{k}\left|P_{k} f\right|^{2}\right)^{1 / 2}\right\|_{\dot{\mathcal{K}}_{2}^{-\alpha, 2}}
$$

A standard estimation gives $\sup _{k}\left\|\chi m\left(2^{k} \cdot\right)\right\|_{\mathcal{L}_{\alpha}^{2}} \leq \sup _{t}\|\varphi m(t \cdot)\|_{\mathcal{L}_{\alpha}^{2}}$. We finish by applying both Littlewood-Paley inequalities in Lemma 3.5.

Proof of Corollary 1.3. Given Theorem 1.2 this is a standard argument. Define $S_{t}$ by $\widehat{S_{t} f}(\xi)=\chi(\rho(\xi) / t) h(\rho(\xi) / t)$ with $h \in B_{\alpha, q^{\prime}}^{2}$. Let $f \in L^{p, q}$. We need to show $\lim _{t \rightarrow \infty} S_{t} f(x)=0$ a.e. on $U_{R}=\left\{x: R^{-1} \leq|x| \leq R\right\}$, for every $R>2$. For this it suffices to show that for every $r>0, \varepsilon>0$ we have

$$
\operatorname{meas}\left\{x \in U_{R}: \limsup _{t \rightarrow \infty}\left|S_{t} f(x)\right|>r\right\}<\varepsilon .
$$

It is easy to see that for any Schwartz function $g$ we have $\lim _{t \rightarrow \infty} S_{t} g(x)=0$ uniformly on compact sets. Let $\sigma=\max \{q, 2\}$. Given any Schwartz function $g$ the left hand side of (32) can now be estimated by

$$
\begin{aligned}
& \operatorname{meas}\left\{x \in U_{R}: \limsup _{t \rightarrow \infty}\left|S_{t}[f-g](x)\right|>r\right\} \\
& \lesssim r^{-2}\left\|\sup _{t>0}\left|S_{t}[f-g]\right|\right\|_{L^{2}\left(U_{R}\right)} \\
& \lesssim r^{-2} R^{d(1 / 2-1 / p)}(\log R)^{1 / 2-1 / \sigma}\left\|\sup _{t>0}\left|S_{t}[f-g]\right|\right\|_{\dot{\mathcal{K}}_{2}^{d\left(\frac{1}{2}-\frac{1}{p}\right), q}} .
\end{aligned}
$$

If we combine Theorem 1.2 with Lemma 3.1 we get

$$
\left\|\sup _{t>0}\left|S_{t}[f-g]\right|\right\|_{\dot{\mathcal{K}}_{2}^{d\left(\frac{1}{2}-\frac{1}{p}\right), q}} \lesssim\left\|h_{\lambda, \gamma}\right\|_{B_{d(1 / 2-1 / q), q^{\prime}}^{2}}\|f-g\|_{L^{p, q}} .
$$

The implicit constants in these estimates are independent of $g$. Since $\mathcal{S}$ is dense in $L^{p, q}$ we may find $g \in \mathcal{S}$ to make the last expression as small as we wish, and (32) follows.

Proof of Theorem 1.1. Let $\chi \in C_{0}^{\infty}$ supported in $(1 / 2,2)$ and equal to 1 in a neighborhood of 1 . We have $h_{\lambda, \gamma}(\rho / t) \widehat{f}(\xi)=\widehat{T}_{t} f(\xi)+\widehat{S}_{t} f(\xi)$ where $T_{t}$ is defined by by $\widehat{T_{t} f}(\xi)=(1-\chi(\rho(\xi) / t)) h_{\lambda, \gamma}(\rho(\xi) / t) \widehat{f}(\xi)$. The maximal function $\sup _{t}\left|T_{t} f\right|$ is controlled by the Hardy-Littlewood maximal function and it is standard that $\lim T_{t} f(x)=f(x)$ almost everywhere for every $f \in$ $L^{r}, r \geq 1$. Next $h_{\lambda, \gamma} \in B_{\alpha, q^{\prime}}^{2}$ for $\alpha=d(1 / 2-1 / p)$ and $\gamma>1-1 / q$, moreover $h_{\lambda, 0} \in B_{\alpha, \infty}^{2}$. By Corollary 1.3, $S_{t} f(x) \rightarrow 0$ a.e. for $f \in L^{p, q}$. This proves the theorem.

\section{The SquARE-FunCtion ESTIMATE}

We now give a proof of Theorem 1.5. Let $\rho \in C_{0}^{\infty}\left(\mathbb{R}^{d}\right)$ be homogeneous of degree 1 , with $\rho(\xi)>0$ for $\xi \neq 0$. We aim to prove, for $1 / 2<\alpha<d / 2$,

$$
\left\|\left(\int_{0}^{\infty}\left|\mathcal{F}^{-1}\left[\frac{\rho^{\beta}}{t^{\beta}}\left(1-\frac{\rho^{\beta}}{t^{\beta}}\right)_{+}^{\alpha-1} \widehat{f}\right]\right|^{2} \frac{d t}{t}\right)^{1 / 2}\right\|_{L^{2}\left(|x|^{-2 \alpha} d x\right)} \lesssim\|f\|_{L^{2}\left(|x|^{-2 \alpha} d x\right)} .
$$


A change of variables shows that (33) implies Theorem 1.5. Let $\eta_{0} \in C_{0}^{\infty}$ be supported on $(-5 / 6,5 / 6)$ and equal to 1 on $(-3 / 4,3 / 4)$. By standard weighted norm estimates for vector-valued singular integrals we have

$$
\left\|\left(\int_{0}^{\infty}\left|\mathcal{F}^{-1}\left[\eta_{0}\left(\frac{\rho}{t}\right) \frac{\rho^{\beta}}{t^{\beta}}\left(1-\frac{\rho^{\beta}}{t^{\beta}}\right)_{+}^{\alpha-1} \widehat{f}\right]\right|^{2} \frac{d t}{t}\right)^{1 / 2}\right\|_{L^{2}\left(|x|^{b} d x\right)} \lesssim\|f\|_{L^{2}\left(|x|^{b} d x\right)}
$$

for all $\alpha>0, \beta>0$ and $-d<b<d$. Thus it suffices to prove

$$
\left\|\left(\int_{0}^{\infty}\left|\mathcal{F}^{-1}\left[\eta_{1}\left(\frac{\rho}{t}\right)\left(1-\frac{\rho^{\beta}}{t^{\beta}}\right)_{+}^{\alpha-1} \widehat{f}\right]\right|^{2} \frac{d t}{t}\right)^{1 / 2}\right\|_{L^{2}\left(|x|^{-2 \alpha} d x\right)} \lesssim\|f\|_{L^{2}\left(|x|^{-2 \alpha} d x\right)}
$$

for $\eta_{1} \in C^{\infty}$ supported in $(3 / 4,5 / 4)$. By Littlewood-Paley theory for the spaces $L^{2}\left(|x|^{-2 \alpha} d x\right)$ and scaling it suffices to prove the analogous inequality in the $t$-localized case for which in the last display the integral over $\mathbb{R}^{+}$is replaced by the integral over $[1,2]$.

We observe also that, with $\eta_{2} \in C_{c}^{\infty}(0, \infty)$,

$$
\frac{\eta_{2}\left(\frac{\rho(\xi)}{t}\right)\left(1-\frac{\rho(\xi)^{\beta}}{t^{\beta}}\right)_{+}^{\alpha-1}}{\left(1-\frac{\rho(\xi)}{t}\right)_{+}^{\alpha-1}}=g_{\alpha}(\xi / t)
$$

where $g_{\alpha} \in \mathcal{L}_{\beta}^{2}$ for all $\alpha>0, \beta>0$. Thus, by an elementary inequality for vector valued operators it suffices to prove that for $1 / 2<\alpha<d / 2$

$$
\left\|\left(\int\left|\mathcal{F}^{-1}\left[\eta(\rho) \varsigma(t)(t-\rho)_{+}^{\alpha-1} \widehat{f}\right]\right|^{2} d t\right)^{1 / 2}\right\|_{L^{2}\left(|x|^{-2 \alpha} d x\right)} \lesssim\|f\|_{L^{2}\left(|x|^{-2 \alpha} d x\right)} ;
$$

here $\varsigma$ is a compactly supported $C^{\infty}$ function. By applying Plancherel's theorem in $t$ the preceding inequality follows from

$$
\left\|\left(\int_{-\infty}^{\infty}\left(1+|\tau|^{2}\right)^{-\alpha}\left|\mathcal{A}_{\tau} f\right|^{2} d \tau\right)^{1 / 2}\right\|_{L^{2}\left(|x|^{-2 \alpha} d x\right)} \lesssim\|f\|_{L^{2}\left(|x|^{-2 \alpha} d x\right)}
$$

with $\mathcal{A}_{\tau}$ as in (17) As before let $\chi_{l}$ be the characteristic function of the annulus $\mathfrak{A}_{l}$. Let $I_{0}=[-1,1]$ and, for $j \geq 1, I_{j}=\left[-2^{j},-2^{j-1}\right] \cup\left[2^{j-1}, 2^{j}\right]$. In order to estimate (34) we make a decomposition which is analogous to the one in (24), and prove

$$
\begin{aligned}
\left\|\left(\sum_{j=0}^{\infty} 2^{-2 j \alpha} \int_{I_{j}}\left|\mathcal{A}_{\tau}\left(\sum_{n \geq-c_{0}} f \chi_{j-n}\right)\right|^{2} d \tau\right)^{1 / 2}\right\|_{L^{2}\left(|x|^{-2 \alpha} d x\right)} & \\
& \lesssim\|f\|_{L^{2}\left(|x|^{-2 \alpha} d x\right)}
\end{aligned}
$$

and

$$
\begin{aligned}
\left\|\left(\sum_{j=0}^{\infty} 2^{-2 j \alpha} \int_{I_{j}}\left|\mathcal{A}_{\tau}\left(\sum_{l \geq j+c_{0}} f \chi_{l}\right)\right|^{2} d \tau\right)^{1 / 2}\right\|_{L^{2}\left(|x|^{-2 \alpha} d x\right)} & \\
& \lesssim\|f\|_{L^{2}\left(|x|^{-2 \alpha} d x\right)} .
\end{aligned}
$$


In order to show (35) we prove for $m \in \mathbb{Z}, n \geq-c_{0}, \varepsilon<\min \left\{\alpha-\frac{1}{2}, \frac{d}{2}-\alpha\right\}$,

$$
\left(\sum_{j=1}^{\infty} 2^{-2 j \alpha} \int_{I_{j}}\left\|\chi_{m} \mathcal{A}_{\tau}\left(f \chi_{j-n}\right)\right\|_{L^{2}\left(|x|^{-2 \alpha} d x\right)}^{2} d \tau\right)^{1 / 2} \lesssim 2^{-|m| \varepsilon} 2^{-n \alpha}\|f\|_{2}
$$

For the proof of (37) let $1<b<d$. The left hand side of (37) can be estimated by

$$
\begin{aligned}
& 2^{-m \frac{2 \alpha-b}{2}}\left(\sum_{j} 2^{-2 j \alpha} \int\left\|\mathcal{A}_{\tau}\left(f \chi_{j-n}\right)\right\|_{L^{2}\left(|x|^{-b} d x\right)}^{2} d \tau\right)^{1 / 2} \\
& \lesssim 2^{-m \frac{2 \alpha-b}{2}}\left(\sum_{j} 2^{-2 j \alpha}\left\|f \chi_{j-n}\right\|_{2}^{2}\right)^{1 / 2}
\end{aligned}
$$

by Proposition 3.4. The last display is estimated by

$$
\begin{aligned}
& 2^{-m \frac{2 \alpha-b}{2}} 2^{-n \alpha}\left(\sum_{j}\left\|f \chi_{j-n}\right\|_{L^{2}\left(|x|^{-2 \alpha} d x\right)}^{2}\right)^{1 / 2} \\
& \lesssim 2^{-m \frac{2 \alpha-b}{2}} 2^{-n \alpha}\|f\|_{L^{2}\left(|x|^{-2 \alpha} d x\right)} .
\end{aligned}
$$

We may choose $b$ such that $1<b<2(\alpha-\varepsilon)$ if $m>0$ and $2(\alpha+\varepsilon)<b<d$ if $m \leq 0$ and then (37) follows.

We sketch the proof of the more straightforward inequality (36) and rely on the argument used for $\mathcal{M}_{k}^{2}$ before. Let $\tilde{\chi}_{l}$ be the characteristic function of $\cup_{-5 \leq i \leq 5} \mathfrak{A}_{l+i}$. Then for $l>j+c_{0}$ and $\tau \in I_{j}$

$$
\left(1-\tilde{\chi}_{l}\right) \mathcal{A}_{\tau}\left(f \chi_{l}\right)(x) \leq C_{N} 2^{-l N} \int \frac{\left|f(y) \chi_{l}(y)\right|}{\left(1+2^{l}|x-y|\right)^{N}} d y
$$

Since $|x|^{-2 \alpha}$ is an $A_{2}$ weight, it is immediate that

$$
\left(\sum_{j=0}^{\infty} 2^{-2 j \alpha} \int_{I_{j}}\left\|\sum_{l>j}\left(1-\tilde{\chi}_{l}\right)\left|\mathcal{A}_{\tau}\left(f \chi_{l}\right)\right|\right\|_{L^{2}\left(|x|^{-2 \alpha} d x\right)}^{2} d \tau\right)^{1 / 2} \lesssim\|f\|_{L^{2}\left(|x|^{-2 \alpha} d x\right)} .
$$

For the main term we use $\alpha>1 / 2$ and estimate

$$
\begin{aligned}
& \left(\sum_{j=0}^{\infty} 2^{-2 j \alpha} \int_{I_{j}}\left\|\sum_{l>j} \tilde{\chi}_{l}\left|\mathcal{A}_{\tau}\left(f \chi_{l}\right)\right|\right\|_{L^{2}(|x|-2 \alpha d x)}^{2} d \tau\right)^{1 / 2} \\
& \lesssim\left(\sum_{j=0}^{\infty} 2^{-2 j \alpha} \int_{I_{j}} \sum_{l>j} 2^{-2 l \alpha}\left\|\mathcal{A}_{\tau}\left(f \chi_{l}\right)\right\|_{2}^{2} d \tau\right)^{1 / 2} \\
& \lesssim\left(\sum_{l>0} \sum_{j=0}^{l-1} 2^{-2 j(\alpha-1)} 2^{-2 l \alpha}\left\|f \chi_{l}\right\|_{2}^{2}\right)^{1 / 2} \lesssim\|f\|_{L^{2}\left(|x|^{-2 \alpha} d x\right)} .
\end{aligned}
$$

Thus (36) is proved. 


\section{REFERENCES}

[1] M. Annoni, Almost everywhere convergence of modified Bochner-Riesz means at the critical index. Dissertation, 2010, University of Missouri.

[2] A. Baernstein, E.T. Sawyer, Embedding and multiplier theorems for $H^{p}\left(\mathbb{R}^{n}\right)$, Mem. Amer. Math. Soc. 53 (1985), no. 318.

[3] A. Carbery, The boundedness of the maximal Bochner-Riesz operator on $L^{4}\left(\mathbb{R}^{2}\right)$, Duke Math. J. 50 (1983), 409-416.

[4] A. Carbery, J.L. Rubio de Francia, L. Vega, Almost everywhere summability of Fourier integrals, J. London Math. Soc. (2) 38 (1988), no. 3, 513-524.

[5] A. Carbery, F. Soria, Almost everywhere convergence of Fourier integrals for functions in Sobolev spaces, and an $L^{2}$ localisation principle, Revista Mat. Iberoamericana 4 No. 2 (1988), 319-337.

[6] M. Christ, On almost everywhere convergence of Bochner-Riesz means in higher dimensions, Proc. Amer. Math. Soc. 95 (1985), 16-20.

[7] L. Cladek, manuscript in preparation.

[8] A. Erdélyi, Asymptotic expansions, Dover Publ. 1956.

[9] G. Garrigós, A. Seeger, Characterizations of Hankel multipliers, Math. Ann. 342, (2008), 31-68.

[10] J. García-Cuerva, J. J. Rubio de Francia, Weighted norm inequalities and related topics. North-Holland Mathematics Studies, 116. Notas de Matemàtica, 104. NorthHolland Publishing Co., Amsterdam, 1985.

[11] J.E. Gilbert, Interpolation between weighted $L^{p}$-spaces, Ark. Mat. 10, 235-249.

[12] C.S. Herz, Lipschitz spaces and Bernstein's theorem on absolutely convergent Fourier transforms, J. Math. Mech. 18 (1968/69), 283-323.

[13] S. Lee, K.M. Rogers, A. Seeger, Improved bounds for Stein's square functions, Proc. Lond. Math. Soc. (3) 104 (2012), no. 6, 1198-1234.

[14] Square functions and maximal operators associated with radial Fourier multipliers, Advances in Analysis: The Legacy of Elias M. Stein, Princeton University Press, 2014, pp. 273-302.

[15] S. Lee, A. Seeger, Square function estimates for the Bochner-Riesz means, in preparation.

[16] B. Muckenhoupt, R. Wheeden, W. Young, $L^{2}$ multipliers with power weights, Adv. in Math. 49 (1983), no. 2, 170-216.

[17] S. Sato, Some weighted estimates for Littlewood-Paley functions and radial multipliers, J. Math. Anal. Appl. 278 (2003), no. 2, 308-323.

[18] A. Seeger, On quasiradial Fourier multipliers and their maximal functions, J. Reine Angew. Math. 370 (1986), 61-73.

[19] _ Necessary conditions for quasiradial Fourier multipliers, Tohoku Math. J. (2) 39 (1987), no. 2, 249-257.

[20] A. Seeger, C.D. Sogge and E.M. Stein, Regularity properties of Fourier integral operators, Annals of Math. 134 (1991) 231-251.

[21] E.M. Stein, On limits of seqences of operators, Ann. of Math. (2) 74 (1961) 140-170.

[22] T. Tao, On the maximal Bochner-Riesz conjecture in the plane for $p<2$. Trans. Amer. Math. Soc. 354 (2002), no. 5, 1947-1959.

[23] M. H. Taibleson, On the theory of Lipschitz spaces of distributions on Euclidean nspace. I. Principal properties. J. Math. Mech. 13 (1964), 407-479.

[24] H. Triebel, Theory of function spaces. Monographs in Mathematics, 78. Birkhäuser Verlag, Basel, 1983. 
Sanghyuk Lee, School of Mathematical Sciences, Seoul National UniverSity, SeOul 15 1-742, Korea

E-mail address: shklee@snu.ac.kr

Andreas Seeger, Department of Mathematics, University of Wisconsin, 480 Lincoln Drive, Madison, WI, 53706, USA

E-mail address: seeger@math.wisc.edu 